\title{
Exclusion of Left Atrial Appendage Thrombus Using Single Phase Coronary Computed Tomography as Compared to Transesophageal Echocardiography in Patients Undergoing Pulmonary Vein Isolation
}

\author{
Jason Saucedo, Shaun Martinho, Dianne Frankel, Ahmad M. Slim, and Robert E. Eckart \\ Cardiology Service, Brooke Army Medical Center, 3551 Roger Brooke Drive, Fort Sam Houston, San Antonio, TX 78234-6200, USA \\ Correspondence should be addressed to Ahmad M. Slim; ahmad.m.slim.mil@mail.mil
}

Received 6 November 2013; Accepted 16 January 2014; Published 20 February 2014

Academic Editors: Y. Khaykin and G. A. Rodriguez-Granillo

Copyright (C) 2014 Jason Saucedo et al. This is an open access article distributed under the Creative Commons Attribution License, which permits unrestricted use, distribution, and reproduction in any medium, provided the original work is properly cited.

Background. Transesophageal echocardiography (TEE) is used for the evaluation of the presence of left atrial appendage (LAA) thrombus prior to pulmonary vein isolation (PVI), while coronary computed tomography angiography (CCTA) is used for anatomic mapping during PVI. Methods. We compared the diagnostic performance of single phase CCTA to TEE in excluding the presence of LAA thrombus in patients undergoing PVI in 172 subjects performed during index hospitalization. Results. The mean age was $51 \pm 13$ years, a median $\mathrm{CHADS}_{2}$ score of $1\left[\mathrm{IQR}_{25,75} 0,1\right.$, range $\left.0-3\right]$ and a mean periprocedural INR of $2.1 \pm 0.6$. The prevalence of an LAA filling defect on single phase CCTA was $9.3 \%$ (6/183) and on TEE was $1.2 \%$ (2/183). Sensitivity, specificity, positive predictive value, and negative predictive value were 100\% (95\% CI, 19.8-100\%), 91.8\% (95\% CI, 94-99\%), 12.5\% (95\% CI, 60-76\%), and 91.8\% ( $95 \%$ CI, 97-100\%) for the detection of LAA filling defect, respectively. Conclusion. Given the utility of a preprocedural single phase CCTA for the performance of PVI, the absence of a filling defect negates the need for a subsequent TEE as an adjunct for exclusion of LAA thrombus.

\section{Background}

Transesophageal echocardiography (TEE) historically is the gold standard for the evaluation of the presence of left atrial appendage thrombus in patients with atrial fibrillation. In a recent study, evaluating spontaneous echo contrast (SEC), left atrial (LA) appendage velocities, and aortic plaque TEE variables in the absence of definitive left atrial thrombus (LA) in five hundred and seventy-nine consecutive patients undergoing pulmonary vein isolation (PVI), all variables with the exception of LA thrombus were not predictive of the occurrence of stroke within thirty days on anticoagulation with Vitamin K Antagonist [1]. This is in contrast to a smaller study of 156 consecutive patients, where functional parameters to include the emptying velocities and SEC were predictive of the presence of LAA thrombus on TEE [2].

Advances in coronary computed tomography angiography (CCTA) imaging have led to high spatial and temporal resolution. CCTA is emerging as a potential screening tool for exclusion of LAA thrombus [3]. Patients routinely undergo TEE before pulmonary vein isolation (PVI) to exclude thrombus and to assess pulmonary venous anatomy and single phase CCTA for incorporation into nonfluoroscopic anatomic mapping systems used during PVI [3].

A number of studies have assessed their application for determining the presence or absence of thrombus. Romero et al. completed a meta-analysis of detection of LAA thrombus by CCTA in patients with atrial fibrillation that demonstrated an overall high accuracy of CCT compared with TEE for the detection of LA/LAA thrombus. The mean sensitivity and specificity were $96 \%$ and $92 \%$ and positive predictive value and negative predictive value were $41 \%$ and $99 \%$ [4].

The purpose of this review is to evaluate the diagnostic accuracy of single phase CCTA obtained routinely in our institution prior to pulmonary vein isolation (PVI) in comparison to TEE in excluding the presence of LAA thrombus 
and thus limiting the need for invasive procedures such as TEE to only patients with possible thrombus on CCTA.

\section{Methods}

2.1. Study Selection. Chart review evaluating concomitant (within 48 hours) single phase 64- or 128-slice CCTA and TEE performed between January 2008 and June 2012 prior to PVI. All procedures were performed in symptomatic individuals with atrial fibrillation over the age of 18 at a single tertiary referral center (San Antonio Military Medical Center, Joint Base San Antonio-Fort Sam Houston, Texas). LAA was evaluated by both CCTA and TEE for filling defects. Imaging modalities were reviewed by a level II echocardiographer and a level III CT imaging staff to assess for the presence or absence of a thrombus.

From January of 2008 to March of 2011, images were obtained using a retrospective helical protocol with a 64-slice CT scanner (Somatom Definition CT, Siemens, Erlangen, Germany). From March of 2011 to March of 2012, studies were obtained utilizing a prospective sequential protocol with $60-$ $80 \%$ image acquisition window. After March of 2012, 128-slice dual head scanner with a single heart beat image acquisition of the complete cardiac anatomy if a heart rate of less than 60 was achieved. (Somatom Definition Flash CT, Siemens, Erlangen, Germany). The subject's EE images were analyzed utilizing Merge Cardio (Merge Healthcare, Chicago, IL) for the evaluation of the entire left atrial appendage. The subject's CCTA images were evaluated using Vitrea 15 software (Vital Images, Inc., Minnetonka, MN) (Figure 1).

2.2. Statistical Analysis. Statistical analysis was performed using IBM SPSS version 19.0 (IBM, Armonk, NY). Continuous variables are presented as means \pm standard deviation and medians with interquartile range, as appropriate. Categorical variables are presented as frequencies with percentages. Comparison of means was performed using one-way ANOVA with post hoc Bonferroni correction, with $P$ values $<0.05$ considered significant.

\section{Results}

We reviewed 403 studies and excluded 231, 15 studies due to time intervals $>48$ hrs between imaging modalities, 60 studies were not included as neither a TEE nor CCTA was done concomitantly. We compared the diagnostic performance of single phase CCTA to TEE in excluding the presence of LAA thrombus in patients undergoing PVI in 172 subjects performed during index hospitalization with a median time between studies of $<24$ hours ( $\mathrm{IQR}_{25,75}$ 0,1 days). statement should read: Mean age was $51 \pm 13$ years with a median CHADS2 score of $1\left(\mathrm{IQR}_{25,75} 0,1\right.$ range $\left.0-3\right)$, and a mean preprocedural INR of $2.1 \pm 0.6$. Prevalence of an LAA filling defect on single phase CCTA was 9.3\% (16/172) versus TEE $1.2 \%(2 / 172)$. The diagnostic accuracy of single phase CCTA is $96.2 \%$. Sensitivity, specificity, positive predictive value, and negative predictive value were $100 \%$ (95\% CI, $19.8-100 \%)$, 91.8\% (95\% CI, 94-99\%), 12.5\% (95\% CI, 60-76\%), and $91.8 \%$
TABLE 1: Baseline demographics of the studied population.

\begin{tabular}{lc}
\hline Age & $51 \pm 13$ \\
HTN & $89(51 \%)$ \\
CHF & $13(7.5 \%)$ \\
CVA & $6(3.4 \%)$ \\
DM & $24(13.9 \%)$ \\
\hline
\end{tabular}

TABLE 2: Sensitivity and specificity as well as positive and negative predictive values with their associated confidence intervals.

\begin{tabular}{lc}
\hline Sensitivity & $100 \%(95 \%$, CI $28.9-100 \%)$ \\
Specificity & $91.8 \%(95 \%$, CI $87.6-95.9 \%)$ \\
PPV & $12.5 \%(95 \%$, CI $2.5-37.5 \%)$ \\
NPV & $91.8 \%(95 \%$, CI $86.5-95.1 \%)$ \\
\hline
\end{tabular}

TABLE 3: Correlations between CCTA and TEE.

\begin{tabular}{lccc}
\hline & Positive TEE & Negative TEE & Total \\
\hline $\begin{array}{l}\text { Positive filling } \\
\text { defect by CCTA }\end{array}$ & 2 & 14 & 16 \\
$\begin{array}{l}\text { Positive filling } \\
\text { defect by CCTA }\end{array}$ & 0 & 156 & 156 \\
\hline Total & 2 & 170 & 172 \\
\hline
\end{tabular}

(95\% CI, 97-100\%) for the detection of LAA filling defect, respectively (Table 1 ).

\section{Discussion}

The study demonstrated that our patient population with atrial fibrillation undergoing PVI may have their LAA excluded with CCTA only. Previous studies on CCTA imaging modalities have already illustrated encouraging results in excluding LAA thrombus. The results of this study demonstrated high sensitivity, specificity, and NPV, that is, $100 \%$, $91.8 \%$, and $91.8 \%$, respectively (Table 2 ). There were a total of 16 positive filling defects on CCTA of which only two were confirmed with TEE. However, of those 156 single phase studies that did not illustrate any defects, all were validated as negative by TEE (Table 3 ).

A recent meta-analysis on detection of LAA thrombus and CT in patients with atrial fibrillation reported sensitivity and specificity of $96 \%$ and $92 \%$, respectively, with a positive and negative predictive value of $41 \%$ and $99 \%$ [4]. The high NPV gives the CCTA modality the potential ability to effectively exclude LAA filling defects. As such, a semiinvasive procedure such as a TEE may potentially be avoided. The absence of a filling defect on CCTA strongly negates the need for a subsequent TEE as an adjunct for exclusion of LAA thrombus.

A major limitation to our study was the absence of discriminating LAA thrombus from spontaneous echo contrast (SEC). CT imaging may identify a filling defect that if viewed by TEE will be assessed to be SEC. Shapiro et al. in their retrospective study detail this finding as a false positive. As stated by Shapiro et al., this issue may be addressed with a delayed scan protocol [5]. Furthermore, this issue can 


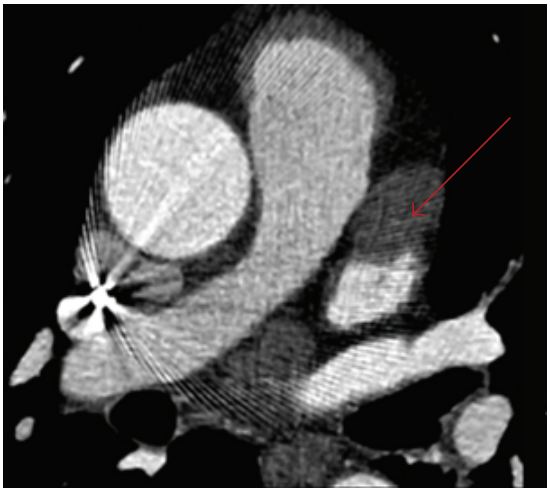

(a)

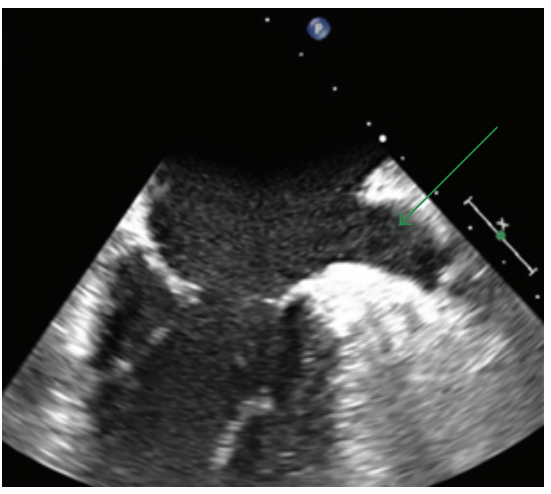

(c)

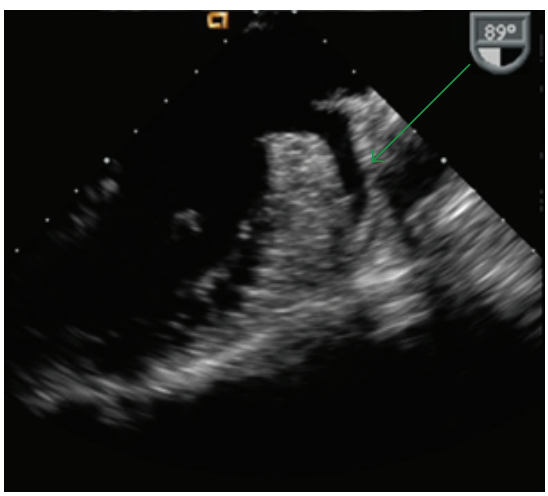

(e)

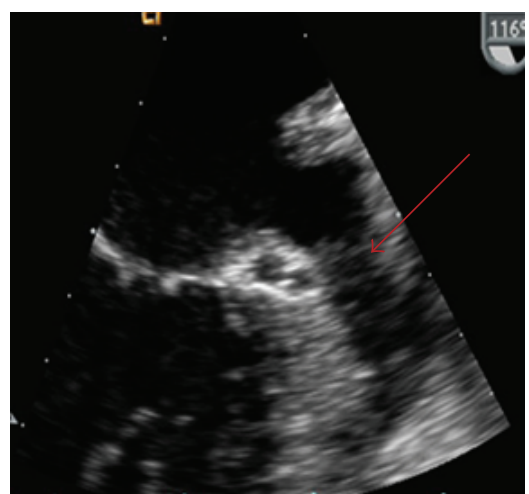

(b)

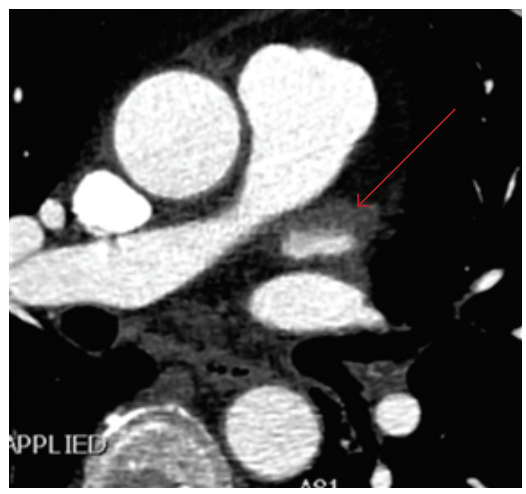

(d)

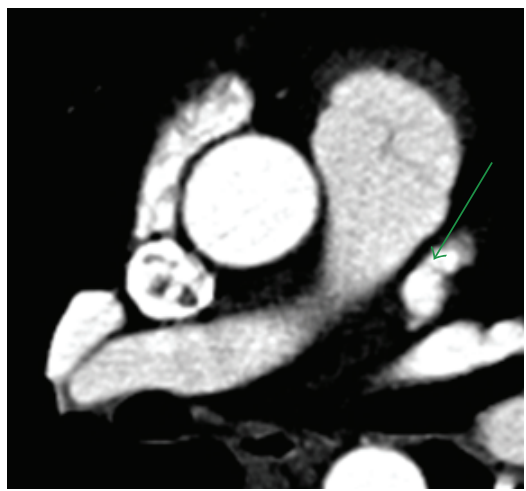

(f)

FIGURE 1: Comparison of CCTA to TEE. Red arrow = filling defect, green arrow = no filling defect. Images (a) and (b) show correlating filling defects as noted on CCTA and TEE in the same subject, while images (c) and (d) show discordance between the two modalities in a different subject, and finally images (e) and (f) demonstrate no defect on either modality as noted by correlating arrows.

further be assessed by using quantitative comparisons of LAA/ascending aorta HU ratios as described by Patel et al. [6]. A study reported a ratio of $\leq 0.75$ as a predictor of LAA thrombus or nonclearing SEC. Other studies having used this ratio to differentiate thrombus from SEC on CCTA, included the categorization of early- and late-phase CT images and comparing thrombus and circulatory stasis and reporting as LAA/AA. Hur et al. evaluated the diagnostic accuracy of a 64slice CCTA for LAA thrombus in stroke patients using TEE as a standard reference standard [3]. The LAA/Asc Ao (early phase) HU ratios of thrombus and circulatory stasis were $0.12 \mathrm{HU} \pm 0.12$ and $0.19 \pm 0.06$, respectively, and LAA/Asc Ao (late phase) $\mathrm{HU}$ ratios were $0.29 \mathrm{HU} \pm 0.12$ and $0.85 \pm 0.12$, respectively.

In a prospective study of 500 consecutive AF ablation procedures performed on 424 patients with a mean age of $55 \pm$ 11 years and a CHADS 2 score mainly between 0 and 1 , similar population to ours, the complications rate reported postablation was $<1 \%$ with zero incidence of death or stroke [7].

\section{Conclusion}

Our findings not only confirms the findings of past studies illustrating the high sensitivity, specificity, and NPV between 
CCTA and TEE in excluding thrombus, but also confirms that a single phase CCTA if part of planned preparation prior to PVI is sufficient to exclude the presence of thrombus. CCTA should be used as an initial step to exclude the presence of filling defects and complimented by invasive procedures such as TEE for confirmation of an LAA thrombus or delayed phase CCTA in the appropriate clinical setting.

\section{Abbreviations \\ CCTA: Coronary computed tomography angiography \\ TTE: Trans thoracic echocardiography \\ TEE: Transesophageal echocardiography \\ ASE: American society of echocardiography \\ PVI: Pulmonary vein isolation \\ HU: Hounsfield units.}

\section{Conflict of Interests}

The authors declare that there is no conflict of interests regarding the publication of this paper. The opinions in this paper of the information contained therein do not constitute endorsement by San Antonio Army Medical Center, the US Army Medical Department, the US Army Office of the Surgeon General, the Department of the Army, the Department of Defense, or the US Government.

\section{References}

[1] K. Chilukuri, S. A. Mayer, D. Scherr et al., "Transoesophageal echocardiography predictors of periprocedural cerebrovascular accident in patients undergoing catheter ablation of atrial fibrillation," Europace, vol. 12, no. 11, pp. 1543-1549, 2010.

[2] M. Iwama, M. Kawasaki, R. Tanaka et al., "Left atrial appendage emptying fraction assessed by a feature-tracking echocardiographic method is a determinant of thrombus in patients with nonvalvular atrial fibrillation," Journal of Cardiology, vol. 59, no. 3, pp. 329-336, 2012.

[3] J. Hur, J. K. Young, H.-J. Lee et al., "Left atrial appendage thrombi in stroke patients: detection with two-phase cardiac CT angiography versus transesophageal echocardiography," Radiology, vol. 251, no. 3, pp. 683-690, 2009.

[4] J. Romero, S. Husain, I. Kelesidis, J. Sanz, H. Medina, and M. Garcia, "Detection of left atrial appendage thrombus by cardiac computed tomography in patients with atrial fibrillation: a meta-analysis," Circulation: Cardiovascular Imaging, vol. 6, no. 2, pp. 185-194, 2013.

[5] M. D. Shapiro, T. G. Neilan, D. S. Jassal et al., "Multidetector computed tomography for the detection of left atrial appendage thrombus: a comparative study with transesophageal echocardiography," Journal of Computer Assisted Tomography, vol. 31, no. 6, pp. 905-909, 2007.

[6] A. Patel, E. Au, K. Donegan et al., "Multidetector row computed tomography for identification of left atrial appendage filling defects in patients undergoing pulmonary vein isolation for treatment of atrial fibrillation: comparison with transesophageal echocardiography," Heart Rhythm, vol. 5, no. 2, pp. 253-260, 2008.

[7] G. Lee, P. B. Sparks, J. B. Morton et al., "Low risk of major complications associated with pulmonary vein antral isolation for atrial fibrillation: results of 500 consecutive ablation procedures in patients with low prevalence of structural heart disease from a single center," Journal of Cardiovascular Electrophysiology, vol. 22, no. 2, pp. 163-168, 2011. 


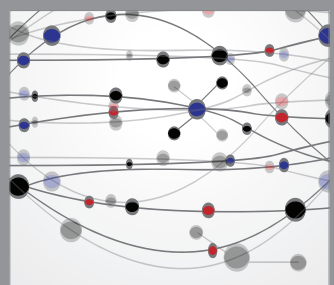

The Scientific World Journal
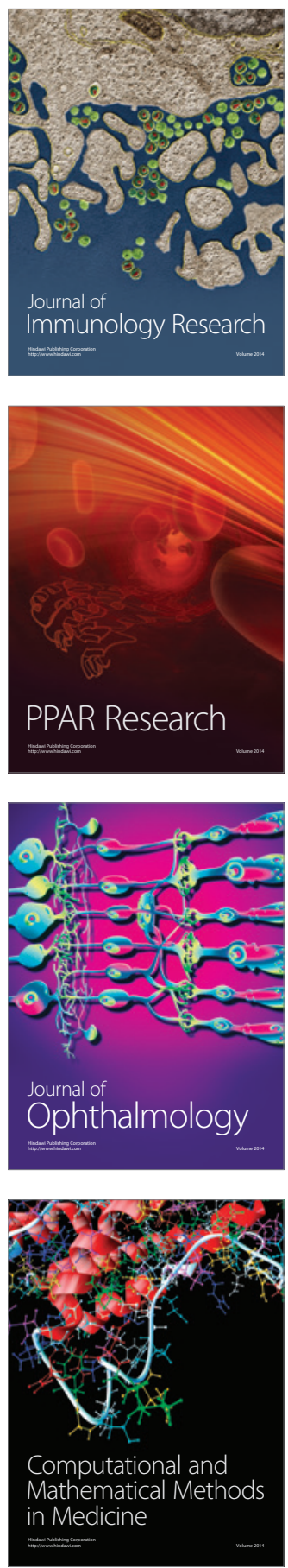

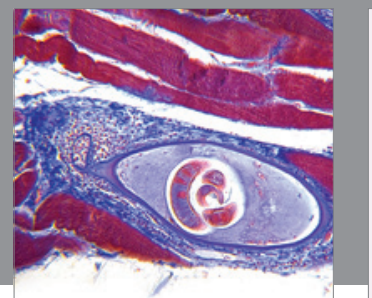

Gastroenterology

Research and Practice
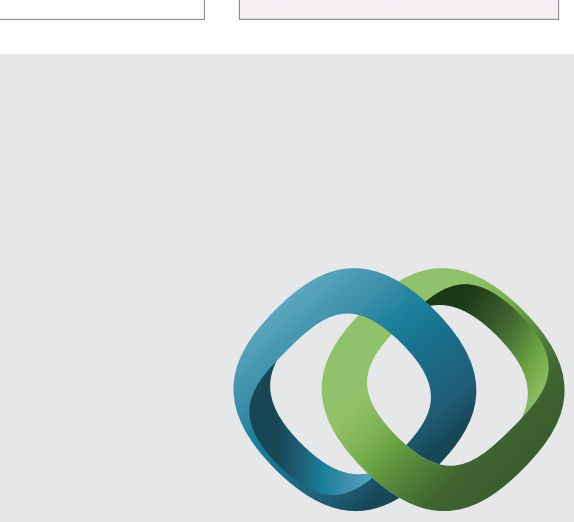

\section{Hindawi}

Submit your manuscripts at

http://www.hindawi.com
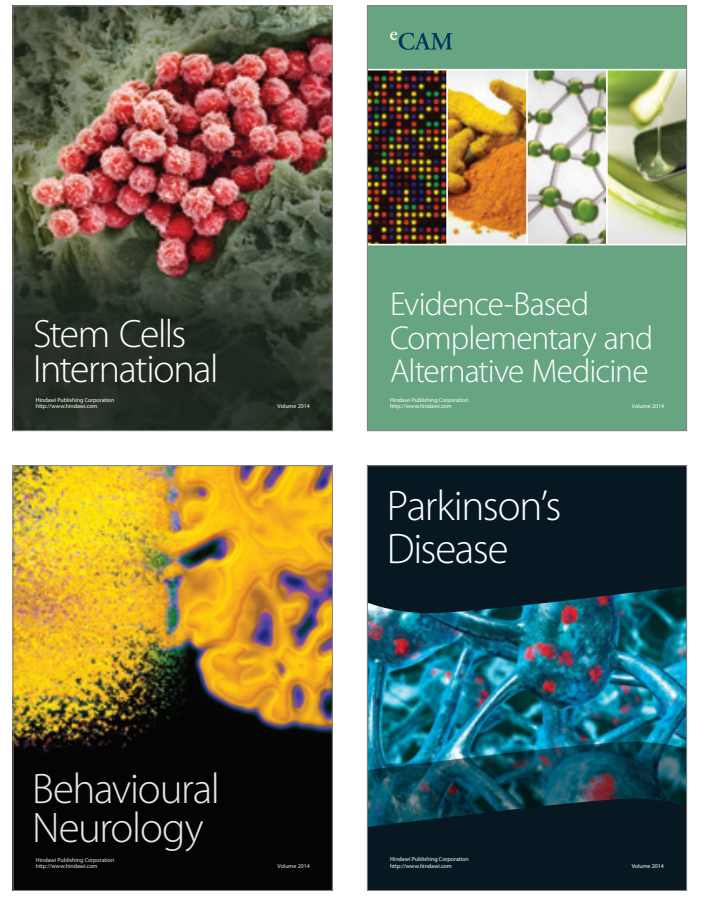
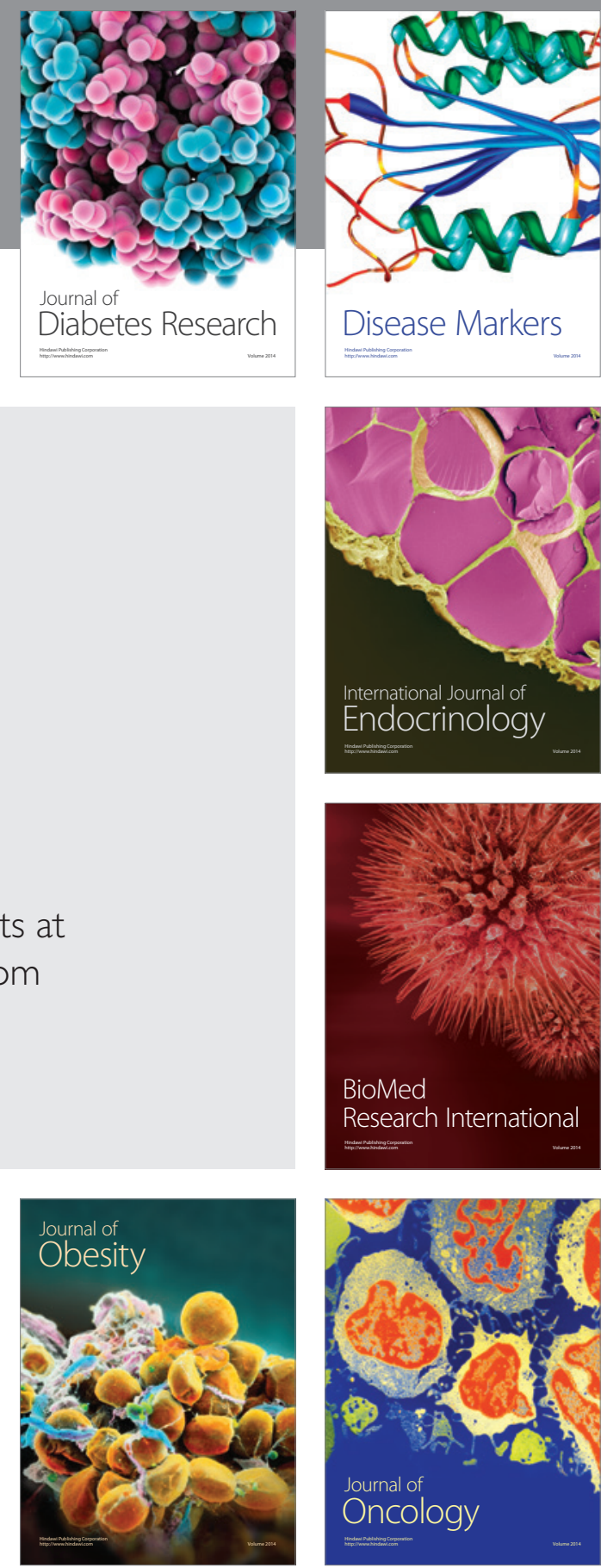

Disease Markers
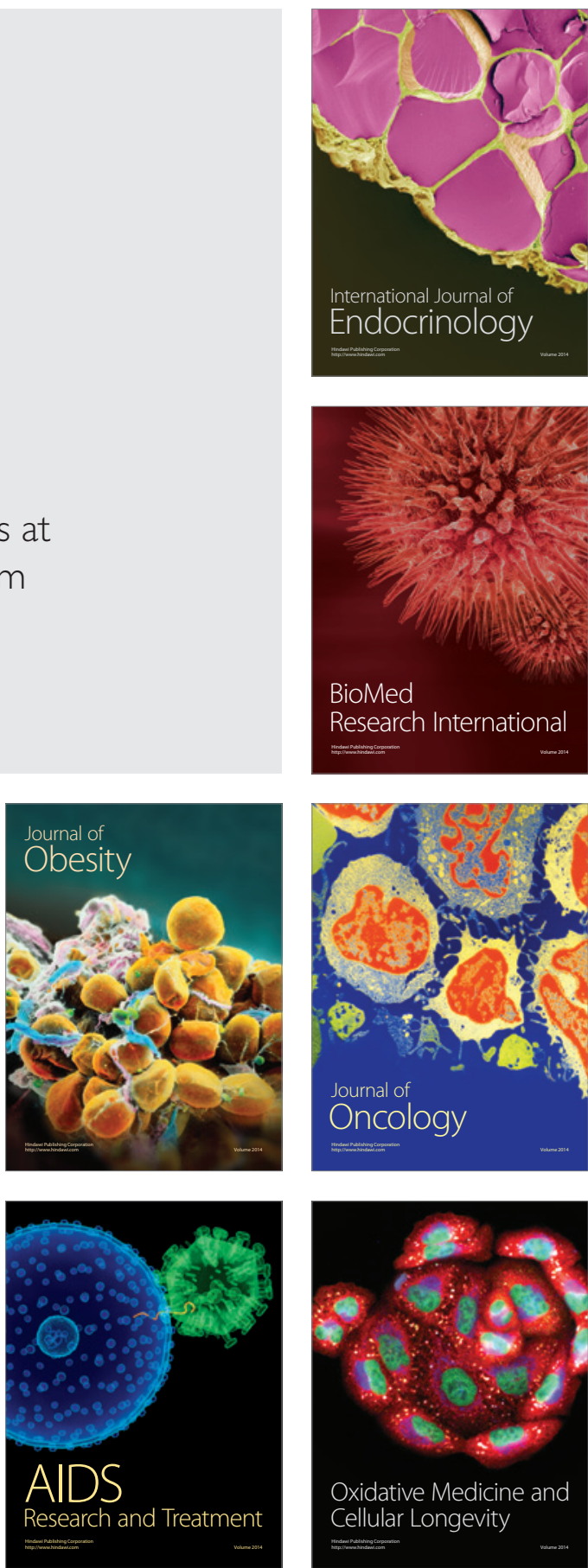